CP, 2021, Vol.10 - No20, pp.35/60, ISSN 2014-6752. Girona (Catalunya). Universitat de Girona. MONTENEGRO GÁLVEZ, D.I.: Cultura, estrategia y crecimiento: un modelo de las personas hacia la aceleración empresarial. Recibido: 01/04/2021 - Aceptado: 03/07/2021

\title{
Cultura, estrategia y crecimiento: un modelo de las personas hacia la aceleración empresarial
}

\section{Culture, strategy and growth: a model of people towards business acceleration}

\author{
AUTOR: \\ Magister Catedrático \\ Diego Ignacio Montenegro Gálvez \\ https://orcid.org/0000-0002-9760-1181 \\ IDE Business School, Universidad Hemisferios \\ diegom@uhemisferios.edu.ec \\ @diegoignaciomontenegro
}

\section{RESUMEN}

Introducción: La pandemia COVID-19 aceleró la presencia el futuro y visibilizó todos los cambios tecnológicos y estratégicos lo que complica aún mas la situación competitiva de las empresas de Ecuador. Metodología: A través de la revisión documental de los principales conceptos referentes a cultura organizacional, estrategia y creación de valor; además, de una investigación profunda realizada en Ecuador, se identifican elementos y elecciones que influyen en la baja competitividad del país y así establecer un modelo para el crecimiento para estas empresas. Resultados: Solamente el $42,4 \%$ de las organizaciones investigadas tienen una creencia profunda para la sostenibilidad; el $42,4 \%$ una propuesta de valor enfocada en la eficiencia y productividad; y, el $62,3 \%$ no cuentan con una metodología para el desarrollo de proyectos de innovación. Discusión: El modelo estratégico utilizado por estas empresas no tiene claridad en el diseño y está enfocado en la eficiencia, los planes operativos y el corto plazo. Conclusiones: El diseño de un modelo cultura -estrategia- crecimiento permite incorporar elementos fundamentales para mejorar el crecimiento y la competitividad de las organizaciones en Ecuador.

\section{ABSTRACT}

Introduction: The COVID-19 pandemic accelerated the presence in the future and made all technological and strategic changes visible which further complicates the competitive situation of companies in Ecuador. Methodology: Through the documentary review of the main concepts referring to organizational culture, strategy and value creation; in addition, to a deep investigation carried out in Ecuador, elements and elections that influence the low competitiveness of the country are identified and thus establish a model for growth for these companies. Results: Only $42.4 \%$ of the organizations investigated have a deep belief in sustainability; $42.4 \%$ a value proposition focused on efficiency and productivity; and, $62.3 \%$ do not have a methodology for the development of innovation projects. Discussion: The strategic model used by these companies lacks clarity in design and is focused on efficiency, operational plans and the short term. Conclusions: The design of a culture -strategy- growth model allows incorporating fundamental elements to improve the growth and competitiveness of organizations in Ecuador. 


\section{PALABRAS CLAVE:}

Cultura empresarial; liderazgo; estrategia; tecnología; competitividad; sostenibilidad.
KEYWORDS: Corporate culture; leadership; strategy; technology; competitiveness; sustainability.

\section{CONTENIDO}

I. Introducción. II. Objetivos. III. Metodología. IV. Resultados. V. Discusión. VI. Conclusiones. VII. Referencias.

\section{CONTENT}

I. Introduction. II. Objectives. III. Methodology. IV. Results. V. Discussion. VI. Conclusions. VII. References.

\section{Introducción}

La pandemia del COVID-19 no produjo un cambio en la sociedad, el cambio ya estaba. Walsh (2019) antes de este evento único, pero posiblemente repetible, mencionaba que el "futuro ya no es la continuación del pasado"; el futuro no es lo que se haga en el futuro, sino lo que se hace en el presente. Entonces, lo que se dejó de hacer en el pasado es lo que nos está afectando actualmente, y define si las organizaciones pueden superar los problemas impredecibles ocasionado por la pandemia con un "momentum" adecuado, con ímpetu, velocidad e impulso necesario (McQueen, 2018).

La década pasada fue de profundos cambios para las organizaciones. La crisis actual no hizo más que adelantar el futuro. Años atrás se habían presentado innegables tendencias como la transformación digital, la computación avanzada y la inteligencia artificial. Ridderstråle y Nordström (2004) dicen que "la tecnología crea oportunidades, y abre posibilidades de vidas más prolongadas y ricas; la tecnología libera, permitiendo a las personas ser ellos mismos si se dispone del capital o de las competencias". Debido a esto, Fisk (2015) afirma que se requieren personas que "cambien el juego empresarial", seres humanos que inspiren, conecten, amplifiquen y hagan posibles las cosas. En un mundo donde la tecnología ha llegado a su punto de explosión, y donde se lleva décadas hablando de la revolución tecnológica producida por un concepto matemático en apariencia complicado y abstracto, pero que es en realidad muy simple y que tiene un efecto real en todo lo que rodea: la curva exponencial (Martínez-Barea, 2014).

Y en esta aceleración como consecuencia de este evento catastrófico que el planeta entero ha vivido, hay que realizarse la pregunta: ¿Por qué las empresas de Ecuador son tan poco competitivas desde hace varios años atrás? Para el World Economic Forum (2018) este país se encontraba en el puesto 86 de 140 países investigados en 2018 en el ranking de competitividad global. El mismo WEForum coloca a Ecuador en la posición 90 para el estudio del año 2019 indicando varias causas para ello como: la falta de una cultura organizacional para mejorar el dinamismo empresarial y la escasa innovación. En el ámbito de la innovación, el Índice Global de Innovación (GIl, por sus siglas en inglés) para el año 2018, reporte respaldado SC Johnson College of Business de la Universidad de Cornell, el INSEAD Business School y el World Intelectual Property Organization (WIPO) coloca a Ecuador en el puesto 97 de 126 países con un puntaje de 26,80 sobre 100 (Dutta, Lanvin, \& Wunsch-Vincent, 2019).

Entonces, ¿qué se debe hacer para mejorar el crecimiento y competitividad de las empresas ecuatorianas? Para esto, se plantea el diseño de un modelo de actuación presente - futuro que incluya la cultura organizacional, creación de valor a través de un modelo de negocio y ejecución de proyectos que logre mejorar el crecimiento empresarial (se puede aplicar también a países con empresas en simi- 
lares condiciones); en una sociedad donde el talento (ahora más que nunca) está interrelacionado por la tecnología y el empleado global desea estar en empresas con amplia libertad junto a líderes que creen un entorno adecuado (Bock, 2015). El cambio abrupto en la forma de trabajar se puede evidenciar en el siguiente estudio sobre el teletrabajo: ${ }^{1}$

\section{LOS NIVELES DE TRABAJO REMOTO SE HAN DISPARADO DURANTE LA CUARENTENA YES PROBABLE QUE SE MANTENGAN ALTOS LUEGO DE LA CRISIS.}

\section{PORCENTAJE DE EMPLEADOS QUE TRABAJAN DE FORMA REMOTAATIEMPO COMPLETO,\%}

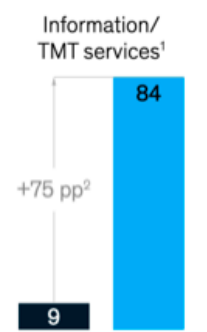

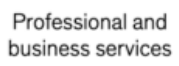

Financial activities

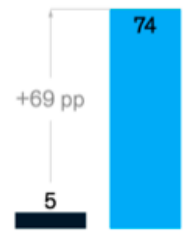

Manufacturing

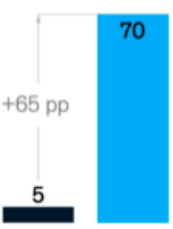

70

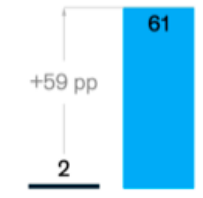

Education and health services

Gráfico 1. Niveles de trabajo remoto durante la cuarenta por el Covid-19.

Fuente: American Time Use Survey y McKinsey Analysis (2020).

La turbulencia en la economía provocada por el Covid-19 es más fácil de soportar para organizaciones que tenían un modelo de actuación con competencias para la diferenciación y la eficiencia; por el contrario, los que pospusieron los cambios en el pasado se encuentran en un presente incierto que va a requerir una nueva forma de pensamiento en liderazgo, visión, valores, innovación, motivación de personas creación de valor y velocidad de reacción. Cualquier modelo en el nuevo paradigma debe simplificar el proceso empresarial y conseguir un alto desempeño, como decía Confucio: "La vida es muy simple, pero las personas insisten en complicarla".2

\section{Objetivos}

El objetivo primario de esta investigación es diseñar un modelo de actuación con los componentes adecuados para conseguir competitividad empresarial.

Adicionalmente, existen unos objetivos secundarios:

- Establecer los elementos de la cultura empresarial que necesitan incorporar las organizaciones de Ecuador y similares en el mundo, para lograr resultados y ser competitivos.

- Realizar las elecciones estratégicas que hacen falta para construir un modelo de negocio capaz de crear y capturar valor para las empresas.

- Determinan la mejor forma de plantear proyectos para conseguir mayor agilidad en la ejecución de las actividades e incrementar la competitividad.

\section{Metodología}

La presente investigación se lleva a cabo a través de una revisión documental de los principales modelos 
y estudios relacionados. Partiendo de datos que se muestran en los siguientes apartados, el presente estudio se enmarca en un tipo de investigación explicativa porque busca la causa del porqué ocurren ciertos sucesos, y permite la comprensión sobre temas específicos y las posibles razones por las que sucede un fenómeno. Es un acercamiento al problema que se pretende estudiar y conocer.

Se trata entonces a través de datos, de tener la comprensión de un tema y distinguir las causas por las que ocurre un fenómeno durante el proceso de investigación para poder anticipar cambios. Esta investigación explicativa debe permitir más adelante replicar estudios para darles mayor profundidad y obtener puntos de vista sobre el fenómeno indagado.

En el estudio motivo de esta investigación, se quiere explicar las posibles causas del estado competitivo de Ecuador (y de sus organizaciones), que en la actualidad está en la parte inferior del ranking de competitividad en lo referente a cultura organizacional, desarrollo estratégico e innovación. Para esto, el estudio se basa en un cuestionario a profundidad realizado por Montenegro, D. I., a finales del año 2019 en 276 empresas de Ecuador con ingresos entre $\$ 5$ millones y $\$ 50$ millones U.S.D. (dólares de Estados Unidos de Norteamérica) en diversas industrias y composición societaria. Para determinar el tamaño de la muestra para el levantamiento de datos se utilizó el muestreo probabilístico aleatorio simple:

$$
n=\frac{Z_{\alpha / 2}^{2} * P * Q * N}{\varepsilon^{2}(N-1)+Z^{2} * P * Q}
$$

Donde:

$n=$ tamaño de la muestra necesaria.

$Z$ = margen de confiabilidad o número de unidades de desviación estándar en la distribución normal que producirá el nivel deseado de confianza (para una confianza de $95 \%$ o un $=0,05, Z=1,96$; para una confianza de $99 \%$ o un $=0,01, Z=2,58$ ).

$P=$ probabilidad que el evento ocurra.

$Q=$ probabilidad que el evento no ocurra.

= error o diferencia máxima entre la media muestral y la media de la población que se está dispuesto a aceptar con el nivel de confianza que se ha definido.

$N=$ tamaño de la población. 


$$
n=\frac{Z_{\alpha / 2}^{2} * P * Q * N}{\varepsilon^{2}(N-1)+Z^{2} * P * Q}
$$

Donde:

$n=$ tamaño de la muestra necesaria (resultado $=208$ empresas para aplicar el cuestionario).

$Z$ = margen de confiabilidad o número de unidades de desviación estándar en la distribución normal que producirá el nivel deseado de confianza (se utiliza $95 \%$ o un $=0,05, Z=1,96$ ).

$P=$ probabilidad que el evento ocurra (se utiliza $50 \%$ ).

$Q=$ probabilidad que el evento no ocurra (se utiliza $50 \%$ ).

= error o diferencia máxima entre la media muestral y la media de la población que se está dispuesto a aceptar con el nivel de confianza que se ha definido (se utiliza 6,5\%).

$N=$ tamaño de la población (se utiliza 2.382 empresas entre $\$ 5$ y $\$ 50$ millones de USD., que fue el último dato reportado en 2018 por la Superintendencia de Compañías del Ecuador).

Al final, se logró conseguir la respuesta de 276 empresas que equivalen a un error de muestreo del $6 \%$.

\section{Resultados}

Muchas organizaciones no logran implementar la estrategia porque no tienen una cultura empresarial alineada y no cuentan con una organización centrada en las personas (Matamala, 2018). La cultura constituye el primer gran elemento del modelo propuesto. Flamholtz (1995) afirma que "la cultura empresarial está compuesta por valores, creencias y normas; los valores se refieren a la importancia que la compañía otorga a los aspectos de calidad, servicio al cliente y trato a los trabajadores. Las creencias son las ideas que las personas dentro de una organización mantienen respecto a ellos mismos y la empresa; y, por último, las normas son las reglas no escritas que guían la forma de interactuar y el comportamiento de las personas". Es así que, la cultura organizacional constituye el sistema operativo de la estrategia centrada en los colaboradores y otros públicos de interés. Andreu y Rosanas (2012) proponen siete pilares para humanizar a la empresa: (1) concebir a la empresa como lo que es, una comunidad de personas; (2) servicio de la persona; (3) volcarse en el aprendizaje y desarrollo personal; (4) pedir una mayor rendición de cuentas de los directivos de la empresa; (5) adoptar una visión holística que respeta la diversidad; (6) reequilibrar los sistemas de retribución; y, (7) aspirar a una misión más elevada.

El primer elemento dentro de la cultura organizacional es el liderazgo consciente con motivación trascendente; es decir, que el líder actúe porque así quiere hacerlo debido al valor que su acción tendrá para otras personas. Kofman (2008) en su modelo de liderazgo incluye actitudes conscientes (responsabilidad incondicional, integridad esencial y humildad ontológica), interacciones conscientes (comunicación au- 
téntica, negociación constructiva y coordinación impecable) y las reacciones conscientes (competencia emocional). En Ecuador, según la investigación realizada a 276 empresas (2019) un 32,2\% de las organizaciones considera que el liderazgo es transaccional con premios y castigos, y un 3,6\% de empresas afirman que no existe liderazgo; consideran también importante al liderazgo transformador (carismático y formador de equipos) con un 33,1\%, mientras que el liderazgo trascendente o consciente tiene una calificación inferior, tal como lo muestra a continuación:

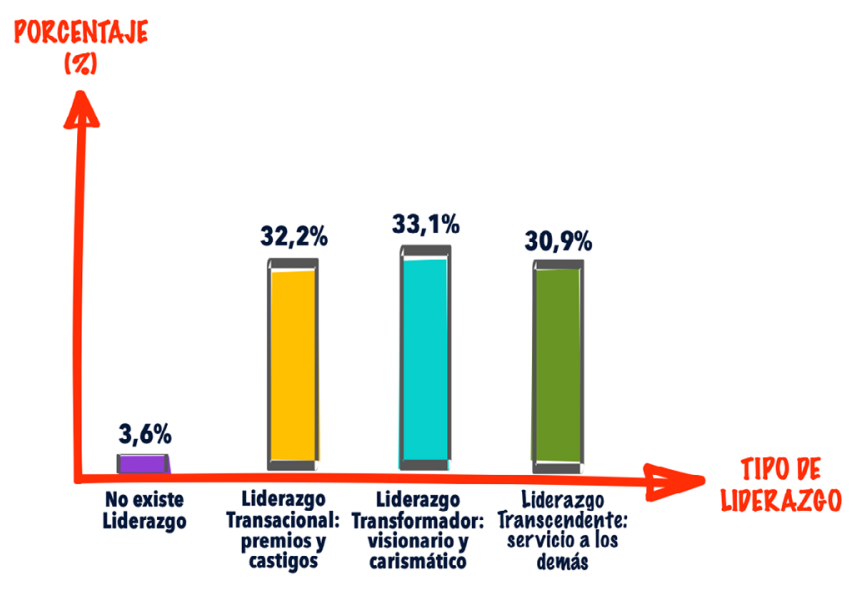

Gráfico 2. Tipo de liderazgo en las empresas de Ecuador.

Fuente: Montenegro, D. I. (2019).

El tipo de liderazgo según el resultado indicado está bastante dividido en las organizaciones. En coherencia con lo anterior, el $48 \%$ de las empresas en Ecuador motivan fuertemente a sus colaboradores con dinero o beneficios materiales, un $41,2 \%$ dan calificaciones bajas a la motivación a través del aprendizaje y conocimiento; 40,1\% dan calificaciones menores a la motivación afectiva; y, un 10\% de los directivos de organizaciones afirman que no existe motivación de ningún tipo. Un 85,8\% muestran que la integridad esencial (reconocer los límites morales y la ética) son críticos para el liderazgo pero que, de acuerdo con los resultados estadísticos de la investigación, "ética y moral" son elementos con menor influencia en los gerentes de todas las variables presentadas.

El segundo elemento de la cultura empresarial es una creencia profunda que va más allá de una visión empresarial con valores, virtudes y ética; como un "ideario" cuyo núcleo son los principios invisibles, incuestionables y difíciles de cambiar. Thompson y Strickland (2004) indican que la principal preocupación de una visión estratégica consiste en saber "hacia dónde vamos" y describe el futuro campo de acción en los negocios de una empresa. García y Dolan (1997) destacan la estrecha relación entre creencias y valores, estableciendo una secuencia de creencias y conductas, siendo las creencias la base de la conducta, de tal manera que puedan modificar la conducta; más que modificar las actitudes, lo que hay que hacer es modificar las creencias y valores.

Sinek (2020) en su último libro "El juego infinito", complementa el concepto de creencia con el de "causa": "Una Causa Justa es una visión concreta de un estado futuro que todavía no existe; es tan atractivo que la 
gente está dispuesta a hacer sacrificios para avanzar hacia esa visión. Puede que no gusten los sacrificios que se hacen, pero se siente que vale la pena hacerlos porque son una Causa Justa". Las empresas con grandes creencias o causas y un sentido del porqué, ignoran a su competencia, mientras que aquellas con un borroso sentido del porqué están obsesionadas con lo que otros están haciendo. Un $42,4 \%$ de las organizaciones investigadas tienen una creencia profunda o trascendente; el 81,2\% dice tener una visión, el $79 \%$ una misión, $79,7 \%$ cuenta con una declaración de valores (así no los practique) y 7,6\% no tienen ningún tipo de declaración de valores.

El tercer elemento de la cultura empresarial son los códigos culturales o "drivers" culturales que incluyen la centralidad en la persona, la búsqueda de unidad (confianza y compromiso), el virtuosismo y las motivaciones humanas. Además, este elemento incluye el enfoque de cualquier organización hacia la innovación (con tecnologías 4.0.), y la responsabilidad social y medioambiental.

"Los negocios sin ética son inhumanos. Para ser verdaderamente humanos, los negocios y la actividad empresarial han de regirse no por la razón de la fuerza, sino por la fuerza de la razón, la cual es precisamente la que fundamenta la ética. La eficiencia no es, pues, ajena a la ética, como tampoco la ética lo es a la eficiencia" (Melé, 1994). José Ramón Pin (2004) complementa lo mencionado por Melé al incluir tres consejos para enfrentarse al nuevo desafío ético de la empresa en el siglo XXI: (1) facilitar el desarrollo de la virtud y no promocionar el desarrollo del vicio; (2) explicar y formar sobre el sentido de la ética; y (3) el ejemplo, el liderazgo ético. Echazarreta y Costa (2019) enfatizan en que ser ético es no dedicarse a actividades especulativas en ningún caso, ni siquiera de forma marginal y extiende el compromiso ético a su cadena de proveedores, distribuidores y clientes; incluso proponen un indicador a través de la medición del número de acciones éticas de proveedores, distribuidores y clientes en comparación con el total de actividades.

Para Pérez-López (2017) se llama unidad de la organización al "comportamiento espontáneo" de las personas que se orienta hacia la puesta en práctica de las acciones determinantes del logro de la eficacia. Es decir, la unidad de una organización expresa el grado de identificación de sus miembros con la eficacia organizacional e indica en qué medida un comportamiento espontáneo viene determinado por el logro de la eficacia en cuanto esta es consecuencia de la satisfacción de las necesidades de otras personas. En Ecuador, un $72,8 \%$ de las organizaciones priorizan las habilidades de las personas para la construcción de la estrategia futura; $26,4 \%$ otorgan una calificación menor a los valores y virtudes de las personas, pero el $87,7 \%$ creen que la estrategia se verá fortalecida si se actúa con ética y moral.

Otro de los "drivers" culturales es la innovación y la tecnología. Para Montenegro et al. (2019), la innovación es: "Una acción, no una cosa, además, es una acción humana. La acción innovadora se ordena desde la inteligencia, no como un acto de azar sino como un proceso razonado de observación, análisis y síntesis, en el que la unidad del pensamiento (la idea) se convierte en la sustancia constitutiva de la innovación". Sin la idea, la innovación no es posible. La idea cultivada en acciones de innovación forma la inventiva humana que, a su vez, constituye la potencia natural para la innovación. La idea entonces parte de la creatividad humana. El modelo que propone Amabile (1988), integra el modelo componencial de la creatividad individual al contexto de trabajo de las organizaciones. Esta teoría describe la influencia de dicho contexto en la creatividad de las personas y los equipos y, a su vez, la influencia de la creatividad de las personas y los equipos en la capacidad general de innovación de las organizaciones. Para Amabile (1988) los elementos de la innovación son: (1) el ambiente organizacional frente a la innovación; (2) recur- 
sos; y, (3) prácticas gerenciales. Además, menciona que los tres componentes del modelo de creatividad son: (1) dominio de conocimientos conocido también como "expertise"; (2) pensamiento creativo; y, (3) motivación intrínseca frente a la tarea.

Creatividad e innovación se ven influenciadas por la tecnología que se mueve cada vez más rápido de lo que la humanidad puede procesar. Durante la siguiente década, se va a experimentar mayor convulsión y creación de riqueza que en los pasados cien años con el impacto de la tecnología en la vida diaria de las personas y en toda la sociedad (Diamandis \& Kotler, 2020). Para Bodell (2018) la tecnología es una fuente de complejidad bastante obvia; los avances tecnológicos fueron concebidos para hacer a las personas y organizaciones más eficientes, y esta afirmación es válida en muchos sentidos; pero sin que las personas se den cuenta se han convertido en máquinas de enviar mensajes electrónicos. La tecnología ha invadido el tiempo libre, el espacio de la familia y el trabajo.

El $72,1 \%$ de las empresas ecuatorianas según Montenegro (2019) creen que la creatividad es muy relevante para la construcción de la estrategia y para 52,6\% la innovación es fundamental. Respecto al componente innovación, el 52,1\% de las organizaciones investigadas dicen poseer un óptimo ambiente organizacional, $47,1 \%$ menciona contar con los recursos y $49,6 \%$ contar con prácticas gerenciales adecuadas para innovar. Sobre la creatividad, $68,1 \%$ responde que su organización tiene alto dominio de los conocimientos técnicos, $60,1 \%$ que las personas cuentan con un pensamiento creativo para explorar nuevas formas de hacer las cosas, y 61,9\% dicen que tienen motivación frente a la tarea y disfrute personal del desafío. Un poco más de la mitad de las empresas en el Ecuador $(52,6 \%)$ piensan que la tecnología es Importante para construir una estrategia consistente a futuro.

Respecto a la Responsabilidad Social Empresarial y en coherencia con los 17 Objetivos de Desarrollo Sostenible (ODS) de las Naciones Unidas, el 61,6\% de las empresas del Ecuador están enfocadas en el ODS 8 "trabajo decente y crecimiento económico", el 34,4\% en el ODS 12 "producción y consumo responsable" y el 32,2\% en el ODS 3 "salud y bienestar". Todas los demás ODS tuvieron respuestas con porcentajes menores al $20 \%$.

El último elemento de la cultura organizacional con centralidad en las personas es el Buen Gobierno Corporativo. Eugenio Marulanda (2017) enfatiza que el gobierno corporativo se refiere a las estructuras organizacionales y de control de las empresas que aseguran la protección de los derechos de los asociados y brindan garantías a los grupos de interés. En términos más simples, se explica como "juego limpio". El gobierno corporativo hace parte de la solución de la corrupción, es la cuota inicial de economías confiables y altamente rentables. Constituye la llave a nuevos clientes, la apertura de nuevos mercados y posibilidades reales de atraer nuevos inversionistas que apalanquen la actividad productiva. Un 10,3\% de las organizaciones investigadas por Montenegro en 2019 dicen contar con un directorio o consejo de administración con miembros independientes, un 9,8\% tiene un protocolo de actuación para la familia propietaria, 18,5\% menciona que no tiene gobierno corporativo con directores independientes o asesores y el $66,3 \%$ de organizaciones cuenta con una junta de accionistas legalmente establecida.

El segundo gran componente del modelo de actuación para la competitividad empresarial tiene relación al modelo de negocio. Ricard (2012) menciona que "el modelo de negocio explica la lógica subyacente de una unidad de negocio, entendida como la forma en que dicha unidad crea y captura valor". Alexander Osterwalder e Ives Pigneur (2011) ratifican el concepto de Ricard y establecen que: "Un modelo de ne- 
gocio describe las bases sobre las que una empresa crea, proporciona y capta valor". Muñoz-Najar y Vilà (2001) complementan la importancia de la creación de valor en un modelo de negocio al decir que "hoy se sabe que los clientes exigen avances en aquellas cosas que valoran. Si valoran un costo menor, lo quieren aun más bajo. Si valoran comodidad y rapidez en el momento de la compra, quieren que esta sea más fácil y efectiva. Si necesitan un consejo experto, quieren que éste sea proporcionado con profundidad, durante un período mayor de tiempo y con trato personalizado". La anterior es claramente la definición de propuesta de valor.

Osterwalder y Pigneur (2011) establecen como herramienta de trabajo un lienzo que permite identificar e introducir innovaciones en el plan de negocios a lo largo del tiempo, aportando claridad sobre la verdadera propuesta de valor de una empresa y su sostenibilidad. Los nueve módulos que componen el lienzo son: (1) propuesta de valor; (2) segmento de cliente; (3) relación que desea establecer con cada segmento de mercado; (4) canales de comunicación, distribución y venta; (5) fuentes de ingresos; (6) actividades clave; (7) recursos clave; (8) asociaciones clave; y, (9) estructura de costos. La síntesis que exige trabajar en el lienzo permite pensar en una empresa en forma global, evitando que el análisis que se atasque en los detalles. Al utilizar un solo gráfico para mostrar el modelo de negocio, se consigue tener siempre presente las distintas relaciones entre los módulos. La sencillez de la metodología facilita la participación y captura de nuevas ideas y propuestas. La figura base del lienzo se muestra a continuación:

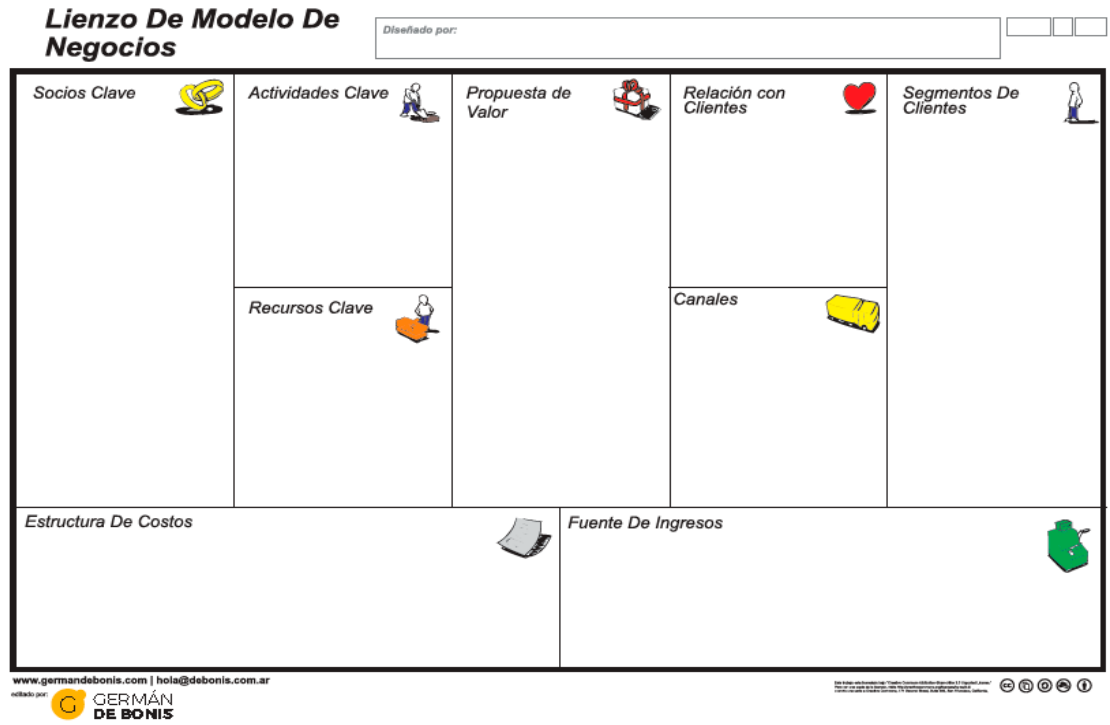

Figura 1. Lienzo Canvas para el diseño de un modelo de negocio.

Fuente: Osterwalder y Pigneur (2011).

Casadesus-Masanell y Ricard (2008) proponen una concepción alternativa del modelo de negocio, definiendo a un modelo de negocio como el "conjunto de elecciones (choices) de la empresa y sus conse- 
cuencias. La estrategia define elecciones que pueden ser políticas, activos físicos y estructuras de gobierno; pero toda elección conlleva consecuencias y estas, a su vez, pueden clasificarse como flexibles o rígidas, según la persistencia de las mismas cuando el efecto de las causas deja de actuar. Lo interesante es que las elecciones y sus consecuencias crean dinamismo y, al final, terminan cerrando el círculo: las elecciones generan consecuencias y estas dan soporte a las propias elecciones (de forma que el ciclo se cierra). Estos círculos pueden ser beneficiosos y se llaman virtuosos; o perjudiciales y se los considera viciosos".

En las contestaciones de la investigación realizada por Montenegro, D. I. (2019) se les pidió a las organizaciones que indiquen las tres elecciones de propuesta de valor (factor que hace que un cliente se incline por una u otra empresa) que más apliquen a su organización. La propuesta de valor enfocada en la "productividad" obtuvo el puntaje más alto con el $42,4 \%$ de las respuestas; la segunda propuesta de valor más utilizada que es "conveniencia" (calidad a un precio competitivo) con el 39,9\% de las contestaciones; $y$, en tercer lugar, la propuesta "simplicidad" (36,6\%) que significa "hacer simple la vida de los consumidores". Otras como "experiencia y servicio" obtuvo el 30,1\% de respuestas e "imagen" el 24,6\%. Todas las demás posibilidades de propuesta de valor tienen porcentajes menores al 19\%.

Respecto a las elecciones sobre el segmento de cliente, consumidor o usuario (los clientes que se van a elegir y aquellos a los que no se quiere), las respuestas conseguidas se muestran en la siguiente tabla:

\begin{tabular}{|c|c|c|}
\hline Elección & Posibilidades & $\begin{array}{c}\text { \% de empresas } \\
\text { (Alto o muy alto uso de la } \\
\text { elección) }\end{array}$ \\
\hline \multirow{6}{*}{$\begin{array}{l}\text { Segmentación de } \\
\text { clientes, } \\
\text { consumidores o } \\
\text { usuarios }\end{array}$} & a) Intuitivamente. & $24,3 \%$ \\
\hline & $\begin{array}{l}\text { b) A través de segmentación dura: edad, } \\
\text { sexo... }\end{array}$ & $38,8 \%$ \\
\hline & $\begin{array}{l}\text { c) A través de segmentación funcional: } \\
\text { atributos, funcionalidades... }\end{array}$ & $61,2 \%$ \\
\hline & d) A través de tribalización emocional. & $18,8 \%$ \\
\hline & $\begin{array}{l}\text { e) A través del enfoque en las creencias } \\
\text { profundas del cliente: valores, normas... }\end{array}$ & $25 \%$ \\
\hline & f) Otras & $7,3 \%$ \\
\hline
\end{tabular}

Tabla 1. Resultado de las elecciones referentes a la segmentación de clientes, consumidores o usuarios.

Fuente: Montenegro, D. I. (2019).

$61,2 \%$ de las respuestas afirman usar segmentación funcional y 38,8\% a través de factores de segmentación "duros" (como geográficos, demográficos o nivel de ingresos). Estos tipos de segmentación pertenecen al esquema tradicional. $24,3 \%$ no tienen una forma de segmentación o lo hacen de manera intuitiva.

En referencia al tipo de relación a establecer con los clientes o consumidores (que son personas), las elecciones que realizaron las empresas de Ecuador se indican a continuación: 
DIEGO IGNACIO MONTENEGRO GÁLVEZ: Cultura, estrategia y crecimiento: un modelo de las personas hacia la aceleración empresarial.

\begin{tabular}{|c|c|c|}
\hline Elección & Posibilidades & $\begin{array}{c}\text { \% de empresas } \\
\text { (Alto o muy alto uso de la } \\
\text { eleceión) }\end{array}$ \\
\hline \multirow{10}{*}{$\begin{array}{l}\text { Forma de } \\
\text { relacionarse de la } \\
\text { organización con } \\
\text { los clientes para } \\
\text { entregarles su } \\
\text { propuesta de valor }\end{array}$} & a) Referencia de otros clientes. & $65,5 \%$ \\
\hline & b) Presentaciones de productos o servicios. & $73,9 \%$ \\
\hline & $\begin{array}{l}\text { c) Cooperación con los clientes para el } \\
\text { desarrollo de una solución. }\end{array}$ & $59 \%$ \\
\hline & $\begin{array}{l}\text { d) Venta de otros productos de la empresa al } \\
\text { mismo cliente. }\end{array}$ & $53,6 \%$ \\
\hline & e) Ofrecer un producto o servicio premium & $64,1 \%$ \\
\hline & $\begin{array}{l}\text { f) Construcción de una relación con el cliente a } \\
\text { través del servicio. }\end{array}$ & $73,9 \%$ \\
\hline & g) Personalización. & $59,4 \%$ \\
\hline & h) Programas de lealtad. & $27,9 \%$ \\
\hline & i) Otras formas de relacionarse con el cliente. & $4,7 \%$ \\
\hline & j) Ninguna de las anteriores. & $4,7 \%$ \\
\hline
\end{tabular}

Tabla 2. Resultado de las elecciones referentes a la relación con los clientes.

Fuente: Montenegro, D. I. (2019).

La respuesta más importante de las organizaciones respecto a este tipo de elecciones de relacionamiento con los consumidores es la presentación de productos o servicios a través de agentes de venta con un $73,9 \%$ de las respuestas, seguida por referencias de nuevos clientes a través de los consumidores actuales $(65,5 \%)$ y ofrecer un producto o servicio premium $(64,1 \%)$.

Los canales de comunicación, distribución y venta establecen el contacto entre empresa y cliente. A la hora de comercializar una propuesta de valor es necesario acertar con la combinación adecuada de canales para llegar a los consumidores. Los canales escogidos por las organizaciones en la investigación de Montenegro, D. I. (2019) son los siguientes:

\begin{tabular}{|l|l|c|}
\hline \multicolumn{1}{|c|}{ Elección } & \multicolumn{1}{|c|}{ Posibilidades } & $\begin{array}{c}\text { \% de empresas } \\
\text { (Alto o muy alto uso de la } \\
\text { elección) }\end{array}$ \\
\hline \multirow{4}{*}{$\begin{array}{c}\text { Canales de venta } \\
\text { de la organización }\end{array}$} & a) Tiendas propias. & $35,8 \%$ \\
\cline { 2 - 3 } & b) Tiendas franquiciadas. & $6,9 \%$ \\
\cline { 2 - 3 } & c) Distribuidores: mayoristas o minoristas. & $37,7 \%$ \\
\cline { 2 - 3 } & e) Fuerza de ventas. & $\mathbf{2 2 , 6 \%}$ \\
\cline { 2 - 3 } & f) Outlets. & $\mathbf{6 5 , 2 \%}$ \\
\hline & g) Máquinas expendendoras. & $\mathbf{4 , 7 \%}$ \\
\hline & h) Otros. & $\mathbf{1 , 8 \%}$ \\
\hline
\end{tabular}

Tabla 3. Resultado de las elecciones referentes a los canales de comunicación, distribución y venta.

Fuente: Montenegro, D. I. (2019). 
Los canales para el contacto entre la empresa y consumidores en las empresas de Ecuador son la fuerza de ventas con el 65,2\% de las contestaciones, tiendas propias físicas con el 35,8\%; y, en tercera posición, los distribuidores mayoristas o minoristas con el $37,7 \%$.

El siguiente grupo de elecciones tienen que ver con la forma de lograr ingresos para la organización contestando a la pregunta ¿qué valor está dispuesto a pagar cada segmento de mercado por la propuesta empresarial? Los resultados de este módulo del modelo de negocio, se muestra en la siguiente tabla:

\begin{tabular}{|c|c|c|}
\hline Elección & Posibilidades & $\begin{array}{c}\text { \% de empresas } \\
\text { (Alto o muy alto uso de la } \\
\text { elección) }\end{array}$ \\
\hline \multirow{8}{*}{$\begin{array}{l}\text { Forma de ingresos } \\
\text { que recibe la } \\
\text { organización por } \\
\text { su operación }\end{array}$} & a) Venta del producto, servicio o solución. & $93,3 \%$ \\
\hline & b) Royalty o comisiones por franquicias. & $5,5 \%$ \\
\hline & c) Comisiones de otro tipo. & $11,6 \%$ \\
\hline & d) Honorarios por asesorías. & $18,1 \%$ \\
\hline & e) Ingresos por publicidad. & $2,9 \%$ \\
\hline & f) Ingresos por proyectos. & $21,8 \%$ \\
\hline & g) Ingresos por suscripciones y membresias. & $5,4 \%$ \\
\hline & h) Otros. & $2,2 \%$ \\
\hline
\end{tabular}

Tabla 4. Resultado de las elecciones referentes a la forma de ingresos que recibe la organización por su operación. Fuente: Montenegro, D. I. (2019).

El 93,3\% de las empresas investigadas reciben ingresos por la venta del producto, servicio o solución que comercializan. Otros ingresos son el resultado de proyectos adicionales que se realizan (21,8\%) y honorarios por asesorías (18,1\%).

Las Actividades clave son las acciones internas que debe emprender una organización para tener éxito. Estas actividades están en función del modelo de negocio y en el caso de las empresas de Ecuador las más importantes se indican a continuación: 
DIEGO IGNACIO MONTENEGRO GÁLVEZ: Cultura, estrategia y crecimiento: un modelo de las personas hacia la aceleración empresarial.

\begin{tabular}{|c|c|c|}
\hline Elección & Posibilidades & $\begin{array}{c}\text { \% de empresas } \\
\text { (Alto o muy alto uso de la } \\
\text { elección) }\end{array}$ \\
\hline \multirow{14}{*}{$\begin{array}{l}\text { Actividades clave } \\
\text { que realiza la } \\
\text { organización para } \\
\text { conseguir la } \\
\text { propuesta de valor }\end{array}$} & a) Optimización de procesos & $70,6 \%$ \\
\hline & b) Innovación de procesos. & $56,5 \%$ \\
\hline & c) Plataforma de servicios. & $37,7 \%$ \\
\hline & d) Plataforma de intercambio de productos. & $11,6 \%$ \\
\hline & $\begin{array}{l}\text { e) Marketing y medios de comunicación online } \\
\text { y offline. }\end{array}$ & $35,5 \%$ \\
\hline & f) Innovación. & $41,7 \%$ \\
\hline & g) Plataforma de software. & $27,2 \%$ \\
\hline & h) Proyectos. & $42,8 \%$ \\
\hline & i) Responsabilidad social empresarial. & $29 \%$ \\
\hline & j) Responsabilidad medioambiental. & $26,4 \%$ \\
\hline & k) Producción y fabricación. & $33,3 \%$ \\
\hline & I) Creación de contenidos. & $17,7 \%$ \\
\hline & m) Entretenimiento y educación & $27,9 \%$ \\
\hline & n) Otros. & $2,9 \%$ \\
\hline
\end{tabular}

Tabla 5. Resultado de las elecciones referentes de actividades clave que realiza la organización para conseguir la propuesta de valor.

Fuente: Montenegro, D. I. (2019).

Los resultados sobre actividades clave que realizan las organizaciones ecuatorianas tienen que ver en primer lugar con los procesos (optimización de los procesos con el 70,6\% de elecciones e innovación de procesos con el $56,5 \%)$. Otras actividades clave que se consideran son: la realización de proyectos $(42,8 \%)$ y contar con innovación $(41,7 \%)$.

Todos los modelos de negocio requieren recursos que permiten a las empresas crear y ofrecer una propuesta de valor para llegar a los mercados, establecer relaciones con los segmentos de mercado y percibir ingresos. En la tabla a continuación se muestran los principales recursos que escogieron las empresas de Ecuador: 


\begin{tabular}{|c|c|c|}
\hline Elección & Posibilidades & $\begin{array}{c}\text { \% de empresas } \\
\text { (Alto o muy alto uso de la } \\
\text { elección) }\end{array}$ \\
\hline \multirow{12}{*}{$\begin{array}{l}\text { Recursos que se } \\
\text { están utilizando en } \\
\text { la organización } \\
\text { para el desarrollo } \\
\text { de la estrategia }\end{array}$} & $\begin{array}{l}\text { a) Recursos financieros: producto de la venta, } \\
\text { préstamos, acciones, obligaciones... }\end{array}$ & $72,9 \%$ \\
\hline & $\begin{array}{l}\text { b) Talento Humano: colaboradores, asesores, } \\
\text { equipo de gerencia, junta directiva... }\end{array}$ & $75 \%$ \\
\hline & $\begin{array}{l}\text { c) Recursos intelectuales: marcas registradas, } \\
\text { patentes, conocimientos, secretos... }\end{array}$ & $38,8 \%$ \\
\hline & $\begin{array}{l}\text { d) Recursos tangibles: edificios, equipos y } \\
\text { localización. }\end{array}$ & $40,5 \%$ \\
\hline & e) Big Data. & $16,7 \%$ \\
\hline & f) Robots y automatización. & $8,3 \%$ \\
\hline & g) Inteligencia Artificial (AI). & $7,3 \%$ \\
\hline & h) Internet de las Cosas (loT). & $10,9 \%$ \\
\hline & i) Impresión 3D. & $3,6 \%$ \\
\hline & $\begin{array}{l}\text { j) Realidad Aumentada (AR) y Realidad Virtual } \\
\text { (VR). }\end{array}$ & $3,2 \%$ \\
\hline & k) Blockchain. & $3,2 \%$ \\
\hline & n) Otras. & $2,1 \%$ \\
\hline
\end{tabular}

Tabla 6. Resultado de las elecciones sobre recursos que se están utilizando en la organización para el desarrollo de la estrategia.

Fuente: Montenegro, D. I. (2019).

Los recursos con más alta elección son el talento humano (75\%); recursos financieros producto de la venta, préstamos y acciones (72,9\%); y, recursos tangibles como edificios, equipos y localización (40,5\%).

Las empresas se asocian por múltiples motivos y estas asociaciones son cada vez más significativas para muchos modelos de negocio. Las empresas crean alianzas para optimizar su estrategia, reducir riesgos o conseguir recursos. Los resultados sobre aliados clave en las organizaciones investigadas se muestran a continuación: 
DIEGO IGNACIO MONTENEGRO GÁLVEZ: Cultura, estrategia y crecimiento: un modelo de las personas hacia la aceleración empresarial.

\begin{tabular}{|c|c|c|}
\hline Elección & Posibilidades & $\begin{array}{c}\% \text { de empresas } \\
\text { (Alto o muy alto uso de la } \\
\text { eleceión) }\end{array}$ \\
\hline \multirow{7}{*}{$\begin{array}{c}\text { Aliados y partners } \\
\text { que trabajan junto } \\
\text { con la } \\
\text { organización }\end{array}$} & a) Aliados para la innovación. & $37,4 \%$ \\
\hline & b) Aliados de recursos compartidos. & $25,3 \%$ \\
\hline & c) Franquiciatarios. & $6,9 \%$ \\
\hline & d) Marketing partners. & $15,2 \%$ \\
\hline & e) Consorcio. & $7,3 \%$ \\
\hline & f) Join Venture o empresa conjunta. & $14,3 \%$ \\
\hline & g) Otros. & $2,6 \%$ \\
\hline
\end{tabular}

Tabla 7. Aliados clave o partners que trabajan junto con la organización

Fuente: Montenegro, D. I. (2019).

Los partners para la innovación son los que reciben la mayor cantidad de respuestas con el (37,4\%), y se refieren en general a proveedores de software o hardware tecnológico, aliados de recursos compartidos $(25,3 \%)$ y marketing partners $(15,2 \%)$.

El último módulo básico del lienzo hace referencia a la estructura de costos que permite una rentabilidad a la empresa. Tanto la creación y la entrega de valor, como el mantenimiento de las relaciones con los clientes o la generación de ingresos tiene un costo que se resta de los ingresos para generar una utilidad. En la tabla siguiente, se muestra un histórico de utilidad generada por las empresas de Ecuador publicado por la Superintendencia de Compañías del Ecuador (2018) en diferentes los principales sectores productivos de la muestra de empresas escogida:

\begin{tabular}{|c|c|c|c|c|c|c|c|c|c|c|c|c|}
\hline \multirow[t]{2}{*}{$\begin{array}{l}\text { ACTIVIDAD DE LA } \\
\text { EMPRESA }\end{array}$} & \multicolumn{3}{|c|}{2018} & \multicolumn{3}{|c|}{2017} & \multicolumn{3}{|c|}{2016} & \multicolumn{3}{|c|}{2015} \\
\hline & $\begin{array}{c}\text { Ingresos } \\
\text { (Miles de } \\
\$ \text { ) }\end{array}$ & $\begin{array}{l}\text { Utilidad } \\
\text { (Miles de } \\
\text { \$) }\end{array}$ & $U / I(\%)$ & $\begin{array}{l}\text { Ingresos } \\
\text { (Miles de } \\
\$ \text { ) }\end{array}$ & $\begin{array}{c}\text { Utilidad } \\
\text { (Miles } \\
\text { de \$) }\end{array}$ & $U / 1(\%)$ & $\begin{array}{l}\text { Ingresos } \\
\text { (Miles de } \\
\$)\end{array}$ & $\begin{array}{c}\text { Utilidad } \\
\text { (Miles } \\
\text { de \$) }\end{array}$ & $\begin{array}{l}\text { ROS } \\
(\%)\end{array}$ & $\begin{array}{l}\text { Ingresos } \\
\text { (Miles de } \\
\$ \text { \$) }\end{array}$ & $\begin{array}{l}\text { Utilidad } \\
\text { (Miles } \\
\text { de \$) }\end{array}$ & U/I (\%) \\
\hline $\begin{array}{l}\text { Distribuidores } \\
\text { mayoristas. }\end{array}$ & 8.713 .302 & 232.739 & $2,67 \%$ & 7.989 .420 & 213.378 & $2,67 \%$ & 8.473 .884 & 115.470 & $1,36 \%$ & 9.384 .420 & 213.344 & $2,27 \%$ \\
\hline Transporte. & 3.500 .410 & 136.637 & $3,90 \%$ & 3.178 .619 & 131.261 & $4,13 \%$ & 3.325.419 & 54.634 & $1,64 \%$ & 3.843 .325 & 163.168 & $4,25 \%$ \\
\hline $\begin{array}{l}\text { Alimentos y } \\
\text { bebidas. }\end{array}$ & 2.886 .262 & 80.776 & $2,80 \%$ & 2.663 .626 & 67.535 & $2,54 \%$ & 2.527 .851 & 64.681 & $2,56 \%$ & 2.662 .690 & 89.285 & $3,35 \%$ \\
\hline $\begin{array}{l}\text { Distribuidores } \\
\text { minoristas. }\end{array}$ & 2.170 .672 & 53.386 & $2,46 \%$ & 1.898 .287 & 54.624 & $2,88 \%$ & 2.116 .792 & 35.498 & $1,68 \%$ & 2.213 .429 & 55.106 & $2,49 \%$ \\
\hline Construcción. & 2.052 .544 & 127.118 & $6,19 \%$ & 2.265 .642 & 151.885 & $6,70 \%$ & 2.497 .383 & 136.428 & $5,46 \%$ & 2.596 .849 & 124.320 & $4,79 \%$ \\
\hline
\end{tabular}

Tabla 8. Utilidad promedio generada en empresas de Ecuador entre $\$ 5$ y $\$ 50$ millones de USD de ingreso divididas por los principales sectores productivos.

Fuente: Superintendencia de compañías del Ecuador (2018). 
La utilidad sobre el ingreso en los diferentes sectores está entre 1,36\% y 6,7\% entre los años 2015 a 2018. Este mismo indicador en otros países de la región es superior al 12\% en promedio.

El tercer y último componente del modelo se refiere a cómo articular y conectar las elecciones de los módulos del modelo de negocio en proyectos innovadores, ágiles y escalables en el tiempo para lograr el crecimiento de la organización. Kotter (2015) asevera que "lo que se necesita es un nuevo y poderoso mecanismo para enfrentar los cambios impuestos por la complejidad creciente y el cambio vertiginoso. La solución que funciona asombrosamente bien (para la ejecución de proyectos) es la implementación de un segundo sistema organizado en forma de red, más como una especie de sistema solar naciente que como una organización madura organizada como si fuera una pirámide de Giza, y diseñado para imprimir agilidad y velocidad. Se trata de un complemento poderoso más que una carga adicional a la jerarquía de la organización madura, a la cual libera para que pueda hacer eficiente a la empresa en el día a día. Este sistema hace que sea más fácil manejar una empresa y a la vez acelera el cambio estratégico. No es cuestión de lo uno o lo otro; es cuestión de ambas cosas: un sistema operativo dual.

Continúa Kotter (2015) diciendo que "la red impulsa el desarrollo de proyectos al permitir que un grupo mucho más grande de personas se conviertan en agentes de cambio para lograr que se haga mucho más y a mayor velocidad, y lo hace también construyendo una urgencia estratégica mucho más contundente, no solamente en un sentido general sino para aprovechar la oportunidad". Este proceso para conseguir los objetivos de un proyecto se convierte en un acelerador para crear y mantener una cultura de agilidad dentro de la organización.

Para desarrollar los proyectos se deben trabajar en equipos multifuncionales, hete-jerárquicos, de varias generaciones y sexos; es en definitiva un equipo que trabaja en un círculo virtuoso generador de valor. Casadesus-Masanell y Ricard (2010) y complementado por Elgar (2012) mencionan que "dentro del conjunto de círculos virtuosos que se generan en un modelo de negocio, tienen especial interés aquel que, integrando aspectos de disponibilidad a pagar, costo, precio y volumen, termina explicando de manera básica la creación y la captura de valor de la empresa. A este círculo virtuoso fundamental que define la unidad de negocio se lo llama value loop". Añaden Casadesus-Masanell y Ricard (2010) que un modelo de negocio entendido bajo esta definición "es tremendamente complejo, ya que hay demasiadas consecuencias y demasiados círculos o loops". El encargado entonces del diseño de la estrategia debe observar la realidad, su complejidad y la manera de entenderla y simplificarla. Se trata entonces de romper los silos departamentales o áreas de la organización para trabajar en proyectos conectados que consigan la ansiada innovación empresarial.

En Ecuador, un 62,3\% de gerentes de las empresas, cuando contestaron al cuestionario de investigación afirmaron que no tienen una metodología para el desarrollo de proyectos tal como indica el siguiente gráfico: 


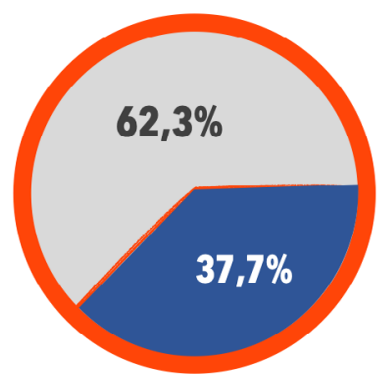

Utiliza una metodología para el desarrollo de proyectos

No Utiliza una metodología para el desarrollo de proyectos

Gráfico 3. Utilización de una metodología para el desarrollo de proyectos

Fuente: Montenegro, D. I. (2019).

El 31,9\% trabajan proyectos empresariales con objetivos compartidos entre varios procesos o áreas funcionales; el 50,3\% dicen que desarrollan los objetivos en cada departamento funcional o proceso individual; $y$, un $10,5 \%$ cree que no tiene objetivos claros o formales. El $40,6 \%$ de las empresas en Ecuador asegura que utiliza indicadores exclusivamente para medir planes operativos; sólo un tercio de las organizaciones dicen utilizar indicadores para medir todo: objetivos, planes y proyectos; un $31,2 \%$ indica que evalúa proyectos estratégicos y $25,7 \%$ añade que no realiza mediciones formales de objetivos, proyectos o planes.

\section{Discusión}

En los primeros años de este siglo, Tom Peters (2003) señalaba: "Se actúa como si el colapso de las empresas "punto com" señalara el final de la Nueva Economía. Pero, de hecho, las organizaciones están al borde de la ola de cambio económico más grande y rentable en mil años. Evitan el fracaso a toda costa y se aferran a ideales como "orden" y "eficiencia". Se debe aceptar el fracaso y gloriarse en la miseria, suciedad y desorden que producen una verdadera innovación". Aún no estamos preparados. Se tiene en primer lugar que entender mejor cómo crecen las organizaciones y por qué muy a menudo estas acaban luchando por su mera supervivencia sin importar el éxito que tuvieron en el pasado, así como también por qué a veces quiebran. Se necesita también comprender mejor por qué unas pocas empresas se recuperan y vuelven a crecer hasta cumplir con su misión de crear buenos puestos de trabajo, servicios y riqueza. Todo ello ayudará a reparar en el papel que la disciplina, la planificación, la fiabilidad y la eficiencia juegan en estos casos, así como el rol que desempeña la pasión, la visión, el compromiso, la velocidad, la agilidad y la cultura (Kotter \& Rathgeber, 2017).

El modelo cultura - estrategia - crecimiento propuesto, pretende mejorar la competitividad de las organizaciones de Ecuador (y similares en el mundo entero); primero, entendiendo las debilidades y elecciones insuficientes en cada uno de los mencionados componentes; y, luego, sugiriendo las elecciones más adecuadas para enfrentar un contexto adverso y demandante en el futuro. Moote (2014) lo mencionaba fuertemente antes de la pandemia: "Estamos de acuerdo en que el mundo cambia rápidamente. El futuro no es como el pasado. El modo en que se hace negocios hoy no será igual a como se hará en el futuro. Resulta tan difícil predecir el clima para los próximos 12 meses como lo es predecir el rendimiento de una empresa", pero hay que intentar hacerlo. 
Los aspectos novedosos e importantes, interpretaciones, hallazgos y análisis de las implicancias de los resultados, se van a detallar en los siguientes apartados por cada uno de los componentes: (1) cultura organizacional; (2) elecciones de modelo de negocio; y, (3) proyectos conectados para el crecimiento.

\section{Cultura organizacional.}

El diseño de cultura empresarial (con centralidad en las personas) que se propone para el modelo se muestra en la siguiente imagen, junto con los resultados más altos en la investigación de Montenegro, D. I. (2019) en empresas de Ecuador en cada uno de sus elementos contituyentes:

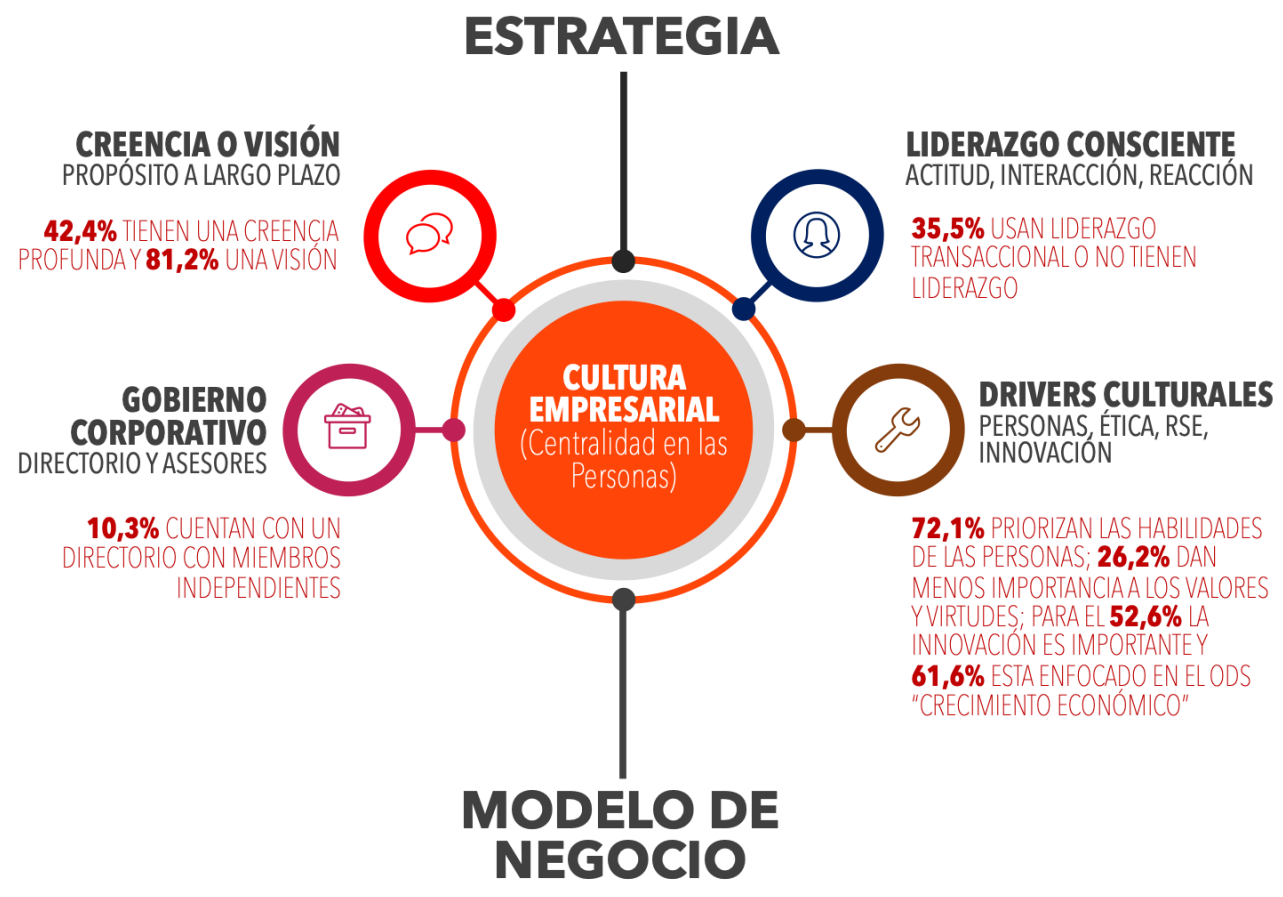

Imagen 1. Componente "cultura empresarial" del modelo propuesto.

Fuente: Montenegro, D. I. (2020).

La discusión se centra en un liderazgo transaccional enfocado hacia la eficiencia y el corto plazo con una motivación material hacia los colaboradores. Para casi un tercio de las empresas investigadas el talento humano no es una prioridad al igual que la ética y los valores; tampoco han desarrollado un claro propósito a largo plazo ni cuentan con el asesoramiento de un directorio o asesores a nivel estratégico. 


\section{Elecciones del modelo de negocio.}

En la siguiente imagen, se muestra el diseño del modelo de negocio para crear valor futuro en la organización con los resultados de las elecciones más relevantes realizadas por las empresas de Ecuador:

\section{CULTURA EMPRESARIAL}

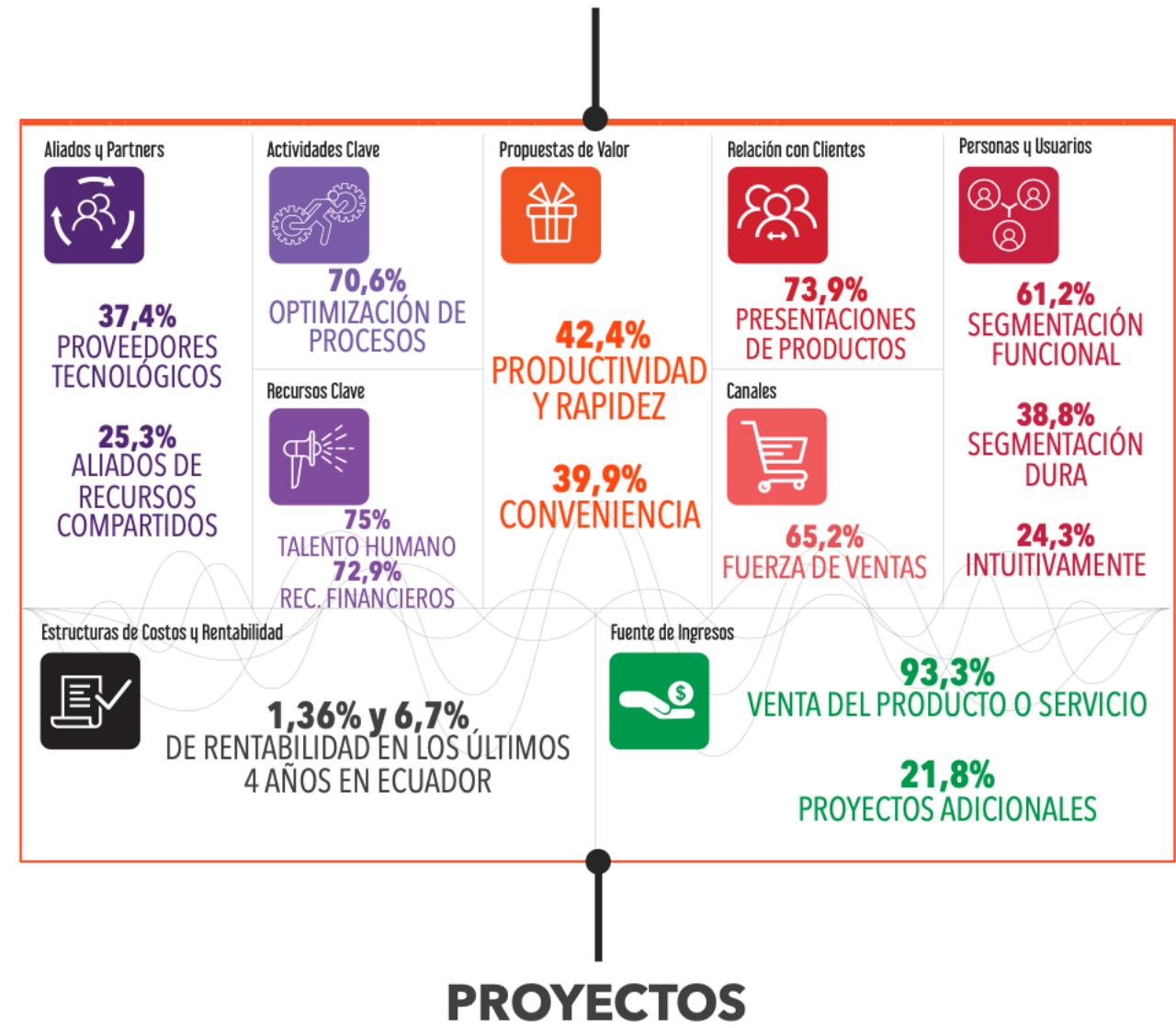

Imagen 2. Componente "modelo de negocio para la creación y captura de valor" en el diseño del modelo propuesto. Fuente: Montenegro, D. I. (2020).

Para lograr una atractividad del mercado, los resultados obtenidos en empresas de Ecuador apuntan a una segmentación insuficiente conectada a una propuesta de valor de productividad y conveniencia en el corto plazo a través de una relación por medio de la fuerza de ventas tradicional, esperando conseguir ingresos de forma mayoritaria por la venta del producto o servicio que la organización comercializa. Para poder conseguir esto, las empresas en el Ecuador piensan en la optimización o cambio de sus procesos internos (enfoque en la eficiencia) financiados a través de la venta del producto y con un talento humano no utilizado en su máxima capacidad; los "partners" no son realmente aliados estratégicos sino más bien proveedores transaccionales de servicios. El resultado final es una baja rentabilidad promedio de las empresas en este país en relación a otras zonas geográficas. 


\section{Proyectos conectados para el crecimiento.}

Es relevante incluir en la discusión la forma cómo se planifican y ejecutan los proyectos resultantes cuando se conectan las elecciones del modelo de negocio. La imagen con el diseño del componente y los resultados obtenidos se muestra a continuación:

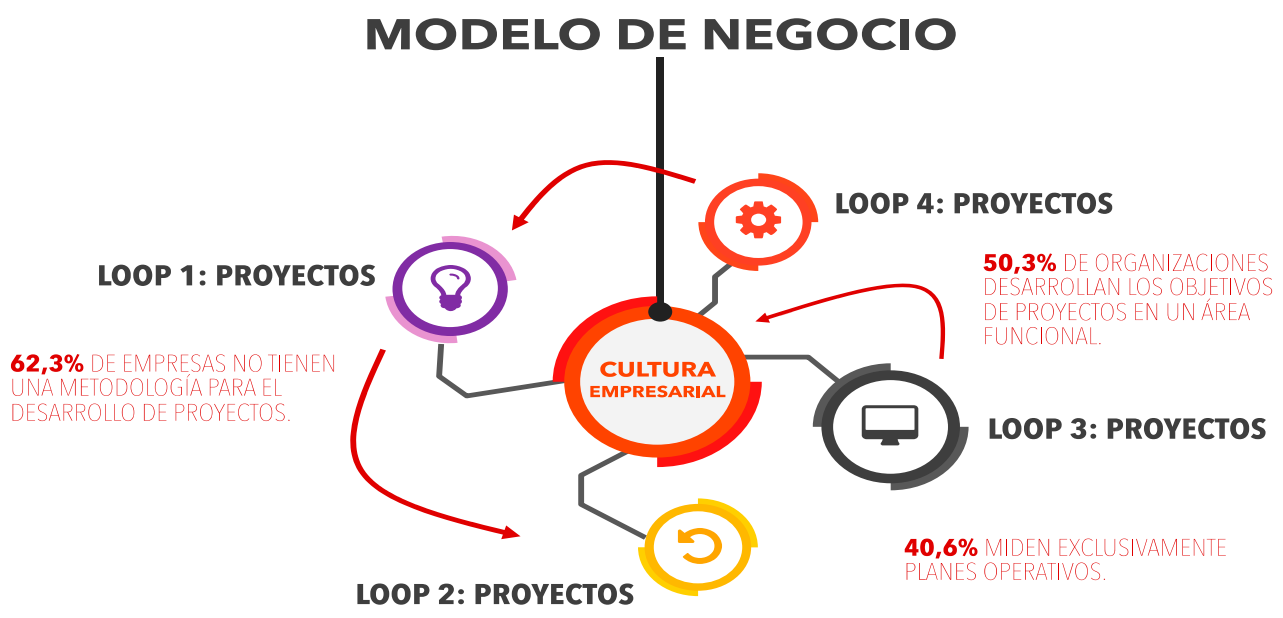

Imagen 3. Componente "proyectos conectados en forma de loop" del modelo de actuación propuesto. Fuente: Montenegro, D. I. (2020).

Los proyectos se priorizan en loops dependiendo de su naturaleza (crecimiento de ingresos, desarrollo de personas, operaciones y tecnología, etc.), conectados unos con otros para lograr un enfoque transversal de la innovación; y, sobre todo, movilizar a la organización para lograr agilidad y velocidad en la práctica. En el caso de las empresas ecuatorianas el enfoque de ejecución es a corto plazo, con trabajo en áreas o departamentos y con planes operativos en lugar de proyectos estratégicos para la sostenibilidad.

\section{Conclusiones}

El negocio, como lo menciona Lucas (1987) es siempre algo específico: "No se dirigen empresas con ideas generales; así lo han hecho muchos de los directivos y se han visto sorprendidos por los detalles concretos, especiales, de cada situación". Las organizaciones se dirigen con modelos sostenibles; ese es justamente el aporte principal de la presente investigación: diseñar un modelo con los componentes específicos para lograr una mayor competitividad empresarial, no solamente para las empresas de Ecuador (ubicado en el puesto 90 de 141 países en el informe de competitividad global) sino para aquellas en cualquier lugar del mundo y que se encuentran en similares circunstancias. Esto se convierte en relevante y notorio en el actual contexto de crisis económica y social causada por el ataque del COVID-19.

El Fondo Monetario Internacional (2020) establece la posibilidad que el decrecimiento mundial en 2020 esté en el orden de: $-8 \%$ a nivel mundial, $-10,2 \%$ en la zona Euro, $-4,7 \%$ en Oriente Medio y Asia central, $-0,8 \%$ en la zona de Asia emergente y en desarrollo, - $-9,4 \%$ en América Latina y el Caribe, y $-3,2 \%$ en el África Subsahariana. Las cifras de recuperación para 2021 son bastante prudentes e impredecibles todavía. 
Tal como se mencionó en el apartado "discusión", el diseño del modelo cultura - estrategia - crecimiento que se propone consta de tres componentes que se muestran completos en la siguiente imagen:

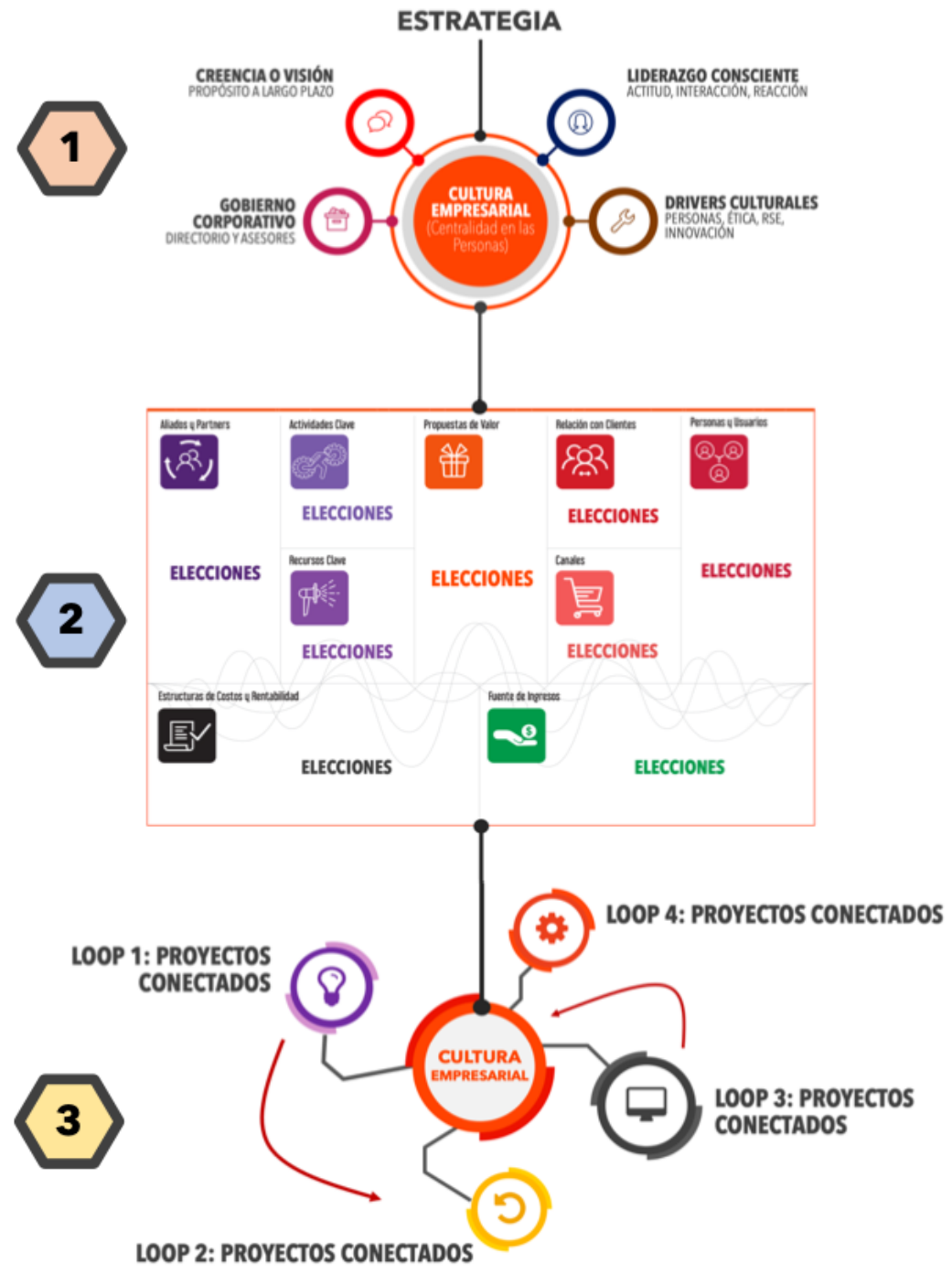

Imagen 4. Modelo de actuación "cultura - estrategia - crecimiento" para la competitividad de las empresas.

Fuente: Montenegro, D. I. (2020). 
En el componente (1) "cultura empresarial", se concluye que las organizaciones requieren a nivel del elemento liderazgo implantar un liderazgo consciente que articule valores, competencias e inteligencia emocional de los líderes a cargo de la estrategia empresarial; la evolución del liderazgo constituye un de los retos empresariales más importantes ahora que los colaboradores están lejos físicamente, en trabajo remoto. Es obligatorio para lograr una mejora competitiva también implementar un propósito a largo plazo en un mayor número de compañías de Ecuador, porque sin su implementación no se tiene una visión clara de crecimiento futuro.

Respecto al driver cultural "innovación", la mitad de organizaciones en Ecuador no consideran la importancia de esta dentro de un modelo estratégico; se requiere, entonces, fomentar el ambiente organizacional hacia la innovación (asumir riesgos, tener una estrategia de innovación e impulsar el entusiasmo de las personas), conseguir los recursos (materiales, profesionales e información) e promover las prácticas gerenciales (objetivos de innovación, planificación de proyectos y compromiso interno). En responsabilidad social las empresas deben buscar otros objetivos de desarrollo más allá del crecimiento económico que impulse la mejora de la sociedad y el mundo. En lo concerniente a Buen Gobierno Corporativo se puede llegar a la conclusión que las organizaciones deben reestructurar su forma de gobierno y aceptar a miembros independientes con nuevas ideas y experiencia; o, permitir asesores "on demand" en caso que no exista la posibilidad de formalizar un consejo de administración.

Las elecciones y sus consecuencias para crear valor son parte del componente (2) del modelo. Del lado de la atractividad del mercado, las empresas para mejorar su competitividad deberían, primero, trabajar en una propuesta de valor fuera de las tradicionalmente usadas como productividad y conveniencia, migrando hacia el desarrollo de propuestas de valor basadas en experiencia del consumidor, diferenciación (contra-corriente) o simplicidad realmente aplicada. El conocimiento del consumidor pasa de la intuición o factores básicos de segmentación a el entendimiento de los principios, valores y emociones que le llevan a elegir una u otra marca. Para que el valor pueda ser trasladado a las personas, la relación que se genere debería ser más profunda procurando la lealtad a través de programas específicos (como la creación de comunidades de clientes). Hoy más que nunca en la era de la pandemia se imponen los canales online (plataformas integrales) como complemento a los canales habituales de comunicación y venta (distribuidores, tiendas propias, página web, redes sociales, software específico y otros). El ingreso que la empresa recibe es el resultado de una aceptación del mercado objetivo y requiere monetizarse más allá de la venta del producto o servicio; una forma de hacerlo es conseguir ingresos a través de servicios complementarios, proyectos adicionales, honorarios o venta de publicidad o información.

Para construir la propuesta de valor las actividades más notables y que no están siendo explotadas a cabalidad por las empresas ecuatorianas son las referentes al diseño de plataformas de producto, servicio, medios y creación de comunidades; si el canal es online, el uso de plataformas tecnológicas permitiría el intercambio eficiente de productos. Las tecnologías exponenciales tienen poca influencia en la generación de competitividad; tecnologías de la era 4.0 como la inteligencia artificial, el internet de las cosas, big data, automatización o blockchain no están siendo utilizadas en la actualidad por las organizaciones investigadas; así como también, las alianzas estratégicas para la innovación, el marketing, las empresas conjuntas o la eficiencia de recursos. Entonces, se concluye, que la baja rentabilidad de estas empresas es debida a la poca diferenciación que afecta el margen de rentabilidad y la ausencia de volumen de venta al no acceder a mercados a través de un desarrollo de plataformas digitales que abarquen nuevos mercados y hábitos de las personas. 
Por último, el componente (3) tiene que ver con la forma de ejecutar los proyectos en las empresas investigadas. Se concluye que la baja competitividad y resultados son producto de la poca utilización de metodologías modernas (como design thinking, scrum, sprint, entre otras). Para poder llevar a cabo proyectos innovadores los objetivos deben ser desarrollados en loops estratégicos con equipos multidisciplinares, hete-jerárquicos, con diferentes edades y conocimientos; sin duda, esto ayuda a la generación de ideas y la velocidad de ejecución rompiendo los "silos departamentales" que sólo son necesarios para resolver los problemas en el corto plazo. La empresa ecuatoriana se enfoca más en planes operativos porque no encuentra una perspectiva de desarrollo a través de un modelo centralizado en la creatividad de las personas para la sostenibilidad a largo plazo.

Este artículo es una aproximación profunda a las sugerencias más visibles para mejorar radicalmente los críticos resultados de competitividad en las organizaciones de Ecuador y es insumo fundamental para la planificación de la estrategia sustentada en una cultura organizacional fuerte. 


\section{Referencias}

Amabile, T. M. (1988). A Model of Organization Innovation. Research in Innovation Behaviour, 10.

Andreu, R., \& Rosanas, J. M. (2012). Manifiesto por una Gestión Empresarial más Humana. IESEInsight, 13-19.

Bock, L. (2015). La Nueva Fórmula del Trabajo. Bogotá: Penguin Ramdom House.

Bodell, L. (2018). Simple. Bogotá: Paidós Empresa.

Casadesus-Masanell, R., \& Ricard, J. E. (2010). From Strategy to Business Models and Onto Tactics. Boston: LRP.

Casadesus-Masanell, R., \& Ricart, J. E. (2008). Competing Through Business Models (A, B y C). Boston: Harvard Business School.

Diamandis, P. H., \& Kotler, S. (2020). The Future is Faster than You Think. New York: Simon \& Schuster.

Dutta, S., Lanvin, B., \& Wunsch-Vincent, S. (2019). WIPO. Obtenido de https://www.wipo.int/edocs/ pubdocs/en/wipo_pub_gii_2018.pdf

Echazarreta, C. A., \& Costa, A. (2019). Estudio y Conceptualización del "Valómetro": un Sistema de Indicadores Empresariales para la Gestión basada en Valores. Revista Latina de Comunicación Social, 74, 573-593.

Elgar, E. (2012). Research in Competitive Strategy. Northampton: G. B. Dagnino.

Fisk, P. (2015). Gamechangers. West Sussex, United Kingdom: Wiley.

Fondo Monetario Internacional. (2020). Informe de Perspectivas de la Economía Mundial. Obtenido de https://www.imf.org/es/Publications/WEO/Issues/2020/06/24/WEOUpdateJune2020

Framholtz, E. G. (1995). Managing Organizational Transitions: Implications for Corporate and Human Resource Management. European Management Journal, 13(1), 39-51.

García, S., \& Dolan, S. (1997). Dirección por Valores. Madrid: McGraw Hill.

Kofman, F. (2008). La Empresa Consciente. Buenos Aires: Aguilar.

Kotter, J. P. (2015). Acelerar. Bogota: Penguim Random House.

Kotter, J., \& Rathgeber, H. (2017). ¡Aquí No Hacemos las Cosas Así! Barcelona: Penguin Random House. 
Lucas Tomás, J. L. (1987). El Proceso de Negocio: Un Modelo de Pensamiento Útil. Barcelona, España: IESE Publising.

Martínez-Barea, J. (2014). El Mundo que Viene. Barcelona: Grupo Planeta.

Marulanda, E. (2017). Es Hora de una Revolución Empresarial. Quito, Ecuador: CAF-BVQ.

Matamala, R. (2018). Organizaciones Coherentes. Bogotá: Paidós Empresa.

McQueen, M. (2018). Momentum. Madrid: Ediciones Urano, S.A.U.

Melé, D. (1994). Ética y Eficiencia Empresarial. Barcelona, España: IESE Publishing.

Montenegro, D. I. (2019). Investigación de Competitividad de la Empresa ecuatoriana en la era 4.0. Quito, Pichincha, Ecuador.

Montenegro, D. I., López, D., \& Susaeta, D. (2019). Epistemology Of Business Innovation, An Approach From The Human Dimension Of The Action. Journal of Multidisciplinary Engineering Science and Technology (JMEST), 9873-9881.

Moote, I. (2014). Design Thinking para la Innovación Estratégica. Barcelona: Urano.

Muñoz-Najar, J. A., \& Vilà, J. (2001). Estrategias de Creación de Valor e Innovación. Barcelona, España: IESE Publishing.

Osterwalder, A., \& Pigneur, Y. (2011). Generación de Modelos de Negocio. Barcelona: Deusto.

Pérez-López, J. A. (2017). Fundamentos de la Dirección de Empresas, 7ma. Edición. Madrid: Rialp.

Peters, T. (2003). Re-imagine! Great Britain: Dorling Kindersley.

Pin, J. R. (2004). Los Nuevos Retos Éticos de la Empresa. Barcelona, España: IESE Publishing.

Ricard, J. E. (2012). Estrategia del Siglo XXI. Modelos de Negocio en Acción. Barcelona, España: IESE Publising.

Ridderstråle, J., \& Nordström, K. (2004). Karaoke Capitalism. Madrid: Pearson Education.

Sinek, S. (2020). El Juego Infinito. Madrid: Urano.

Superintendencia de Compañías del Ecuador (2018). Obtenido de https://appscvs.supercias.gob. ec/rankingCias/rankingCias.zul?id=S\&tipo $=1$.

Thompson, A. A., \& Strickland, A. J. (2004). Administración Estratégica, 13va Edición. México D. F.: McGraw-Hill Interamericana. 
Walsh, M. (2019). The Algorithmic Leader. Canada: Amanda Lewis.

World Economic Forum. (2019). Obtenido de The Global Competitiveness Report 2019: http:// www3.weforum.org/docs/WEF_TheGlobalCompetitivenessReport2019.pdf

\section{CURRICULUM VITAE}

\section{Diego Ignacio Montenegro}

PHD (c) en Economía y Empresa por la Universitat de Girona. Top Manager Harvard University. Varias maestrías en Dirección Empresarial. Programas de innovación en Singularity University. Miembro del Directorio en empresas de diferentes sectores en Ecuador. Consultor y Asesor. Speaker Internacional. Presidente de Emotionshare Corporation. Gerente General de la Universidad Hemisferios de Ecuador. Profesor del IDE Business School. Coautor del libro EmotionShare, artículos científicos, casos de estudio, publicaciones y creador del Modelo CulteX para estrategia empresarial. 